\title{
A Patient with Trisomy 15 and Subglottic Tracheal Granuloma: Anesthetic Management for Emergency Rigid Bronchoscopy
}

\author{
Sergey Pisklakov, Vincent Cordero, Vanny Le \\ Department of Anesthesiology and Perioperative Medicine, New Jersey Medical School, University of Medicine and Dentistry of \\ New Jersey, Newark, USA. \\ Email: pisklase@umdnj.edu
}

Received October 20 ${ }^{\text {th }}, 2012$; revised November $25^{\text {th }}, 2012$; accepted December $15^{\text {th }}, 2012$

\begin{abstract}
Trisomy 15 is a rare genetic disorder presenting unique anesthetic challenges. This is a case of a patient with Trisomy 15 and unrepaired ventriculo-septal defect presented for emergent removal of a subglottic tracheal granuloma. Developed anesthetic plan allowed the patient to breathe spontaneously with a combination of inhalational and intravenous anesthetics. Our technique offered optimal operating conditions and adequate depth of anesthesia. The important points of the case include an understanding of the physical characteristics of a patient with Trisomy 15 and unrepaired ventriculo-septal defect and an anesthetic plan for emergent removal of an obstructing subglottic granuloma via rigid bronchoscopy.
\end{abstract}

Keywords: Trisomy 15; Difficult Airway; Anesthesia for Rigid Bronchoscopy; Ventriculo-Septal Defect

\section{Introduction}

Trisomy 15 is a rare genetic disorder that can present unique challenges for anesthesiologists. Characteristic features of this disorder may include craniofacial abnormalities, growth and developmental delay, mental retardation, feeding difficulties, gastroesophageal reflux disease (GERD), muscular spasticity, scoliosis, and cardiac abnormalities [1]. This is a case of a patient with Tri- somy 15 complaining of severe dyspnea, presented for emergent rigid bronchoscopy and removal of a subglottic tracheal granuloma.

\section{Case}

The patient was a 16-year-old female diagnosed with Trisomy 15 early in life who presented with severe dyspnea, for emergent subglottic granuloma removal. Medical history included GERD, spastic quadriparesis, severe kyphoscoliosis, unrepaired ventriculoseptal defect (VSD) with possible right-to-left shunt and developmental delay. Surgical history included dental extractions, tracheostomy at age 1 , and tracheostomy revision at age 12 . The patient had no known allergies. Her medications included levalbuterol and budesonide. Review of systems was unremarkable except for dyspnea. Vital signs were within normal limits. Physical exam revealed clear lungs, mild inspiratory stridor, holosystolic murmur on auscultation, and gastrostomy tube in place. She was awake but non-verbal. She had a size 5.0 Shiley tracheostomy in place. After a discussion with the surgeons, the anesthetic plan was to keep the patient spontaneously breathing through the tracheostomy, with intermittent removal of tracheal canula during periods of surgical instrumentation via rigid bronchoscope from above. A 22-gauge IV line was placed in her right foot. Sevofurane $1.5 \%$ was delivered via tracheostomy tube. Intravenous midazolam $0.5 \mathrm{mg}$ and ketamine (in incremental doses) were also given. Right radial $22 \mathrm{G}$ arterial catheter was inserted. Total dose of ketamine was $80 \mathrm{mg}$ titrated over $15 \mathrm{~min}$ utes, maintaining spontaneous ventilation and normal blood pressure. The tracheostomy tube was then removed, and a rigid bronchoscope was used to visualize the granuloma that was causing the subglottic airway obstruction. The granuloma was seen on the anterior wall of the trachea Figure 1, and was removed with optical forceps. After confirmation that the obstruction had been relieved, the tracheostomy tube was replaced. The patient tolerated the procedure well and emerged from anesthesia without complication. She was brought to the post anesthesia care unit in stable condition.

\section{Discussion and Conclusion}

There were numerous anesthetic challenges presented in this case. This patient with Trisomy 15 had characteristic physical features of the disease, including craniofacialabnormalities including a small mandible, GERD, 


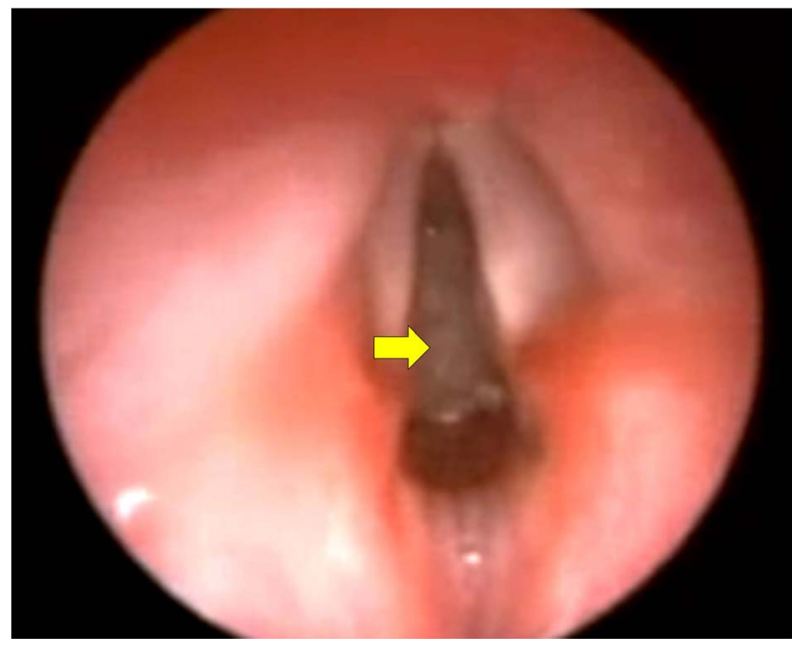

Figure 1. Subglottic granuloma.

muscular spasticity, scoliosis, and an unrepaired VSD, which is one of the most common forms of congenital heart defect in children. VSD may be perimembranous and due to muscular septum defects. Endocardial-cushion-type VSDs are less common and often associated with AV valve insufficiency [2]. In patients with moderate to large defects, symptoms become evident in the first few weeks of life. When shunt is small, patients remain asymptomatic, they grow and develop normally. With time, the septal leaflet of the tricuspid valve may become adherent to the ventricular septal surface, spontaneously closing the perimembranous VSD. Similarly, a muscular VSD may close spontaneously with progressive compression of the defect by the growing myocardial tissue. Endocardial-cushion defects and outlet defects do not close without intervention [3]. Untreated, these infants with high pulmonary blood flow and high pulmonary artery pressures will present with congestive heart failure: poor growth, rapid/labored breathing, tachycardia, and diaphoresis. If they survive without therapy, they develop pulmonary vascular disease and progress to Eisenmenger's physiology [4].

These features raised concerns such as difficulty achieving peripheral intravenous access, full stomach precautions, restrictive lung disease, potential right-to-left shunt, and difficult airway management despite the patient having a tracheostomy. The subglottic granuloma precluded the use of an endotracheal tube or jet ventilation, as the granuloma could have been dislodged further into the trachea or bronchus. Dislodgement could also have caused bleeding, which would further complicate airway management such as difficulty ventilating, oxygen desaturation, bronchospasm, and suboptimal operating conditions $[5,6]$. By first inducing the patient with sevoflurane through the tracheostomy, we were able to attain intravenous and intra-arterial access. Ketamine provided supplemental anesthesia during periods of tracheal decanulation, while maintaining spontaneous respirations as well as systemic vascular resistance (SVR) in order to minimize right-to-left shunt. Continuous positive pressure ventilation affects the intrathoracic to extrathoracic vascular pressure gradients, such that return of blood flow to the right ventricle is impaired and pulmonary vascular impedance is increased, resulting in enhanced right ventricular after load. The combination of both mechanisms is believed to represent the major determinants for the depression of cardiac output during mechanical ventilation. The periodic reduction of intrathoracic pressure resulting from maintained spontaneous breathing during mechanical ventilatory support promotes the venous return to the heart and right- and leftventricular filling, thereby increasing cardiac output and $\mathrm{O}_{2}$ transport capacity [7]. Maintaining spontaneous respirations was obviously advantageous in this case.

Midazolam was given to provide amnesia and to reduce the psychotropic effects of ketamine. This technique provided optimal conditions for the ENT surgeon to perform the procedure successfully.

The important points of the case include an understanding of the physical characteristics of a patient with Trisomy 15 and an anesthetic plan for emergent removal of an obstructing subglottic granuloma via rigid bronchoscopy. Our anesthetic plan allowed the patient to breathe spontaneously with a combination of sevoflurane, midazolam, and ketamine. The ketamine allowed us to maintain the patient's SVR. This technique offered optimal operating conditions and adequate depth of anesthesia.

\section{REFERENCES}

[1] J. Roggenbuck, et al., "Duplication of the Distal Long Arm of Chromosome 15: Report of Three New Patients and Review of the Literature," American Journal of Medical Genetics, Vol. 126A, No. 4, 2004, pp. 398-402. doi:10.1002/ajmg.a.20617

[2] B. Bruneau, "The Developmental Genetics of Congenital Heart Disease,” Nature, Vol. 451, No. 7181, 2008, pp. 943-948. doi:10.1038/nature06801

[3] D. Penny and V. Wesley, "Ventricular Septal Defect," Lancet, Vol. 377, No. 9771, 2011, pp. 1103-1112. doi:10.1016/S0140-6736(10)61339-6

[4] R. Sommer, et al., "Pathophysiology of Congenital Heart Disease in the Adult. Part I: Shunt Lesions,” Circulation Vol. 117, 2008, pp. 1090-1099. doi:10.1161/CIRCULATIONAHA.107.714402

[5] C. Derkay, "Recurrent Respiratory Papillomatosis,” Laryngoscope, Vol. 111, No. 1, 2001, pp. 57-69. doi10.1097/00005537-200101000-00011

[6] X. Shen and W. Li, "Anesthetic Management for an Infant Undergoing Endoscopic Resection of a Large Subglottic Papilloma," North American Journal of Medical Sciences, Vol. 1, No. 2, 2009, pp. 83-85.

[7] D. Grinnan and J. Truwit, "Clinical Review: Respiratory Mechanics in Spontaneous and Assisted Ventilation,” Critical Care, Vol. 9, 2005, pp. 472-484. doi:10.1186/cc3516 